\title{
IntellWheels: intelligent wheelchair with user-centered design
}

\author{
Marcelo R. Petry \\ and Antonio Paulo Moreira \\ INESC TEC and Faculty of Engineering \\ University of Porto, Portugal \\ \{marcelo.petry, amoreira\}@fe.up.pt
}

\author{
Brigida Monica Faria \\ ESTSP, Polytechnic Institute of Porto \\ and IEETA, University of Aveiro \\ Aveiro, Portugal \\ btf@estsp.ipp.pt
}

\author{
Luis Paulo Reis \\ LIACC, University of Porto and \\ DSI/EEUM, University of Minho \\ Guimares, Portugal \\ lpreis@dsi.uminho.pt
}

\begin{abstract}
Intelligent wheelchairs can become an important solution to assist physically impaired individuals who find it difficult or impossible to drive regular powered wheelchairs. However, when designing the hardware architecture several projects compromise the user comfort and the wheelchair normal usability in order to solve robotic problems. In this paper we describe the main concepts regarding the design of the IntellWheels intelligent wheelchair. Our approach has a user-centered perspective, in which the needs and limitations of physically impaired users are given extensive attention at each stage of the design process. Finally, our design was evaluated through a public opinion assessment. A statistical analysis suggested that the design was effective to mitigate the visual and ergonomic impacts caused by the addition of sensorial and processing capabilities on the wheelchair.
\end{abstract}

\section{INTRODUCTION}

Nowadays, society is more and more concerned to promote the social inclusion of impaired individuals. For that, mobility plays an important role, since the amount of independence of a person is closely related to how independently mobile they are. In addition to independence and self-esteem, some studies reveal that mobility can have positive psychosocial and cognitive development of physically disabled children [1].

Physically impaired people often rely upon assistive devices such as wheelchairs, crutches, canes, and artificial limbs to increase, maintain, or improve their functional capabilities. However, a generalization of the treatment and assistance strategies is hardly achieved since each patient shows a different combination of symptoms and levels of motor control. Another constraint is that, in many cases, motor disabilities come associated with cognitive and sensorial impairment, which often lead to driving/navigational problems even when motor impairments are not severe. Therefore, there is a growing demand for intelligent and safer assistive devices. To accommodate users who find operating standard mobility devices difficult or impossible, researchers have used technologies originally developed for mobile robots to create Intelligent Wheelchairs (IW)[2].

According to Fehr et al. [3], 91\% of the clinicians believe that wheelchairs with automated navigation systems can be useful at least for a few users, and $23 \%$ believe the systems can be useful for many of them. Another study, conducted by Simpson et al. [2], estimated that between $61 \%$ to $91 \%$ of all the wheelchair users would benefit somehow from the features of intelligent wheelchairs. Therefore, investment in research and commercialization of intelligent wheelchair have much greater potential impact than previously thought.

Powered wheelchairs are typically composed by a metal frame with four wheels and a seat, batteries, two motors, one motor controller and joysticks. Such configuration is adequate to act in the environment with the constant supervision of a human operator. However, it does not allow the wheelchair to perform higher level tasks. To be considered minimally intelligent, an wheelchair needs to sense its surroundings and react according to changes in the environment, user commands and goals. Therefore, the standard wheelchair configuration needs to be complemented with additional sensors, control electronics and computational hardware [4]. In IntellWheels, this additional set of metal frames and electronic devices are referred to as hardware framework.

When designing the intelligent wheelchair hardware architecture most projects concern, in fact, with solutions to robotic problems. Such solution-centered designs tend to disconsider the typical wheelchair users and their limitation. These wheelchair indeed present a desired feature, or perform better in some situations, but may also create inoperable sophisticated wheeled devices (at least for individuals with limited mobility). It is not hard to find designs that assemble laser scanners in the region between the user legs, bumpers close their feet, sonar rings in from of seat and poles over the patients heads.

In this paper we propose a user-centered hardware design, in which the needs and limitations of physically impaired users are given attention. Impaired individuals spends a significant part of their life on their wheelchair, thus user's comfort is regarded as a main priority. The outline of the paper is the following. Section II presents relevant related works in the area of intelligent wheelchairs. Section III presents to the reader a description of the project IntellWheels. Section IV addresses our user-centered hardware design. Section $\mathrm{V}$ presents the results of the public opinion assessment regarding the visual appearance of the IntellWheels prototype, and Section VI the conclusions of this research.

\section{RELATED WORK}

Since the 1980's, several projects of intelligent wheelchairs proposed alternatives to assist individuals with mobility impairments. Smartchair, for example, is an intelligent wheelchair designed to navigate autonomously to a given destination, 
navigate in the hallway, navigate through doors and manual navigation with obstacle avoidance [5]. The Mobile Internet Connected Assistant (MICA) project from the EISLAB, spans in the area from navigation techniques to the design aspects of intelligent wheelchairs [6]. SENA is one of the few IW projects that concerns with the communication system between wheelchair modules. The project is based on a multi-agent system, which facilitates communication and mechanisms for redundancy [7]. Through its multi-agent system, ACCoMo [8] claims to be able to provide an autonomous navigation with obstacle avoidance, a cooperative behaviour with other wheelchair and a collaborative behaviour with the user. The system intelligence is given by reinforcement learning, neuronal networks and genetic algorithms. FRIEND is composed by a powered wheelchair and a robotic manipulator, which provides the ability to grasp and manipulate objects [9].

RoboChair aims to develop a high performance low cost intelligent wheelchairs, and is focused an intelligent control system and a friendly user interface for voice control, emotion and gesture recognition [10]. The MIT intelligent wheelchair project proposes to enhance ordinary powered wheelchairs and focus on the development of an speech interface to interpret and follow natural language directions [11]. Sharioto proposes a shared control that attempts to estimate the user's intent from user's noisy input signal (a joystick) and the interaction with the perceived environment to generate navigational behaviors [12]. The intelligent wheelchair prototype from the University of Shiga [13] propose an obstacle avoidance algorithm based on neural networks, varying the connection weights of the neural network according to the distance to obstacles in the vicinity of the wheelchair. RobChair [14] is steered with voice commands and assisted by a reactive fuzzy logic controller. The prototype presents a distributed modular architecture, and provides autonomous and semi-autonomous navigation with obstacle avoidance. Vulcan, from the University of Texas Austin [15] focuses on motion control, ARTY [16] focuses on developing an intelligent pediatric wheelchair, and the project from the University of Zaragoza [17] that focus on mobile robot navigation and brain-computer interfaces.

\section{OVERVIEW OF THE INTELLWhEELS PROJECT}

Despite the several projects under development, there was not a flexible model to easily convert a regular powered wheelchair into an intelligent wheelchair. Usually, these projects have rigid hardware and software architectures, are cost prohibitive for most potential users and typically requires a very difficult configuration. In an attempt to address some of these issues, we proposed the project IntellWheels [4]. The focus of the project is to develop an intelligent wheelchair with flexible multimodal interface that facilitates the development and test of new methodologies and techniques, and whose integration into commercially available powered wheelchairs may be performed with only minor modifications.

IntellWheels has an intelligent wheelchair simulator (IntellSim). IntellSim enables easier and more precise data gathering, high control over the environment, is an important pedagogically technique for training and allows the interactions between the real and virtual objects [18]. Since the physical disability is a very wide concept and the symptoms specific to each individual, it is important to provide the largest possible number of input methods in order to assist individuals with different capabilities. Thus, in addition to the traditional joystick, the user has the option to use a game joystick, keyboard, touch-screen display, head gestures input, facial expressions, voice. Finally, the integration of a commercial brain computer interface that recognizes facial expressions and thoughts is been tested, but due to its low accuracy it is still very difficult to use these device to enable safe and robust commands to the intelligent wheelchair [19]. An evaluation of the distinct input methods available to control the wheelchair is presented in [20].

IntellWheels has a multi modal interface (MMI), designed to allow the simultaneous connection of several input devices. Since this is a system that aims to be used by disabled people, safety is of extreme importance. In order to avoid the potential accidents caused by the false recognition of user commands, the MMI allows the user define sequences of inputs, which are subjected to a reliability test [21]. Such sequences are be composed of inputs from the same input device (homogeneous inputs) or from different input devices (heterogeneous inputs). Thus, users can define the most suitable input sequences taking into account to their limitations. Each input sequence can than be associated to one of the actions that the wheelchair is capable to perform. The unique combination between the heterogeneous input sequences and their flexible association with wheelchair actions provide the user the capability to create its own communication language with the wheelchair.

IntellWheels navigation system is responsible for performing the wheelchair's sensors treatment, localization and driving the wheelchair between different locations. The user control module is the application in which the user defines the type and parameters that the controller will use for automatic mode. After choosing one of seven types of actions (following the line, point, the angle, following the left wall, the right wall, wait, stop) several parameters and configuration fields become available to the user. The localization system is based on dead reckoning techniques [22]. IntellWheels multi-level control architecture is subdivided into three layers: strategic layer (goal planning and path planning), tactical layer (control of basic actions, and linear and angular speeds) and basic control layer (control of wheel speeds). A goal planner was implemented with Planning Domain Definition Language - PDDL. The system than generate a path in order to achieve the objectives proposed by the planner, taking into account information from the world model. To find a path from a given initial point to a given goal point, the system has an adapted $\mathrm{A}^{*}$ Algorithm implemented. Later, the tactical layer of the control module subdivide the path into basic forms (lines, circles, points), and computing the wheelchair's linear and angular speeds to put the wheelchair into motion. Finally, the lowest level of control (Basic Control Layer) converts linear and angular speeds into wheel speeds send them through serial communication to the interface board [23].

\section{USER-CENTERED HARDWARE FrAMEWORK DESIGN}

Since the addition of any element to the wheelchair may become a nuisance, our proposed design avoided bulky and heavy sensors, and paid special attention to place all components out of the user work space. Only solutions that does not interfere with the normal wheelchair operation are 


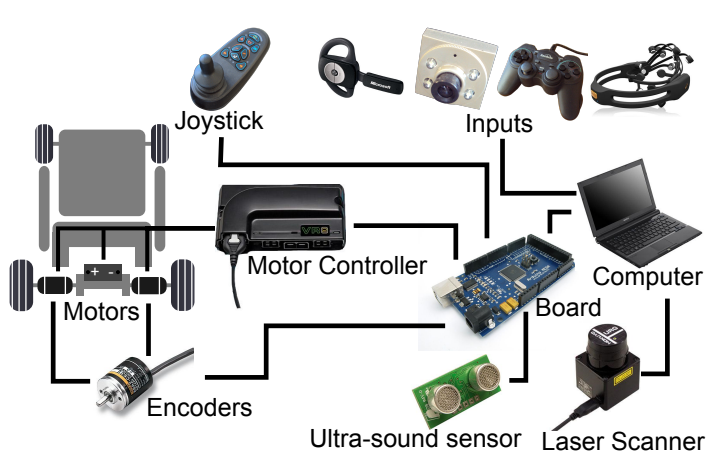

Fig. 1: Architecture of the IntellWheels hardware framework.

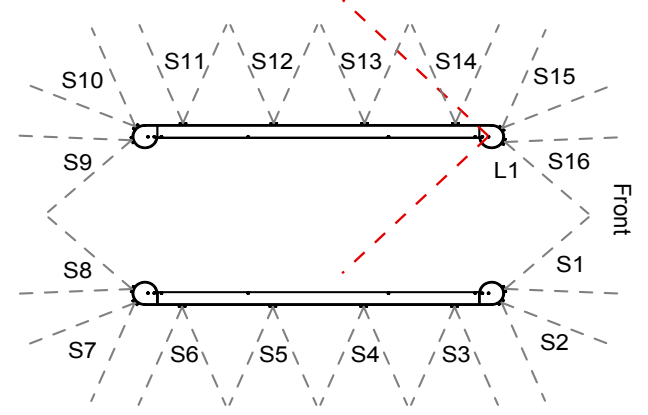

Fig. 2: Placement and FOV of the sensors.

implemented. The proposed design also seeks to reduce the visual impact of the hardware framework, and maintain its compatibility with multiple wheelchair brands and models. An intelligent wheelchair system that requires substantial modification may be impractical for installation in many of the wheelchair models currently available on the market, interfere with normal service of the wheelchair, and prevent potential users from obtaining wheelchairs that could provide mobility assistance. For this reason, we propose a modular system that can be added to a variety of commercial power wheelchairs with minimal modifications.

The architecture of the framework and its connections with the original wheelchair system are depicted in the Figure 1. A computational unit (Intel core 2, 1.2GHz, 2GB RAM) runs the multi-agent system that controls the intelligent wheelchair. A laser scanner, with a field of view of 270 , provides to the computer unit distance measures with high accuracy, which in the future can be used for mapping and localization. Two encoders coupled directly in the motor shaft are connected to the interface board and provide information about the wheel revolutions, that in turn are used to estimate the wheelchair displacement and relative localization. Sixteen ultra-sound transducers, with a field of view of 45 , are connected to the interface board and provide raw distance information that is used by the obstacle avoidance algorithm to prevent collisions. An interface board process information from the encoders and the ultra-sound sensors and send them to the computer unit. The interface board also receives the reference speed of each wheel from the computer unit and generates the corresponding analogical signal that is sent to the motor controller. Power for the sensors and interface board is drawn from the wheelchair batteries though a voltage regulator.

The framework is "inserted" into a power wheelchair control system between the user's input device and the wheelchair

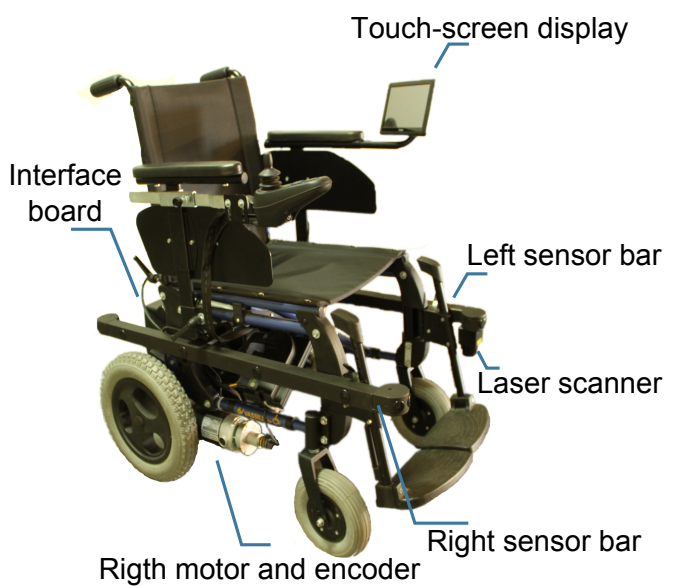

Fig. 3: Components of the IntellWheels prototype.

motor controller. Since most of wheelchair motor control uses a proprietary version of CAN bus, intercepting the joystick signal requires opening the joystick module, reading the wires that carry the joystick signal, and altering the signal to those wires. To avoid the need to open the joystick module, it would be necessary to have access to the bus protocol, or use specific motor controllers that accept signals from external devices.

Normally, the input device is plugged directly into the motor controller. When the framework is installed, however, both the input device and the motor controller are connected to the interface board. The interface board reads the signal from the input device and sends a revised signal to the wheelchair motor controller. The motor controller then treats the revised signal as if it came directly from the input device. Under normal circumstances in which the user operates the wheelchair manually, the revised joystick signal is identical to the original signal. But if an obstacle is detected, the collision avoidance algorithm alters the joystick signal to avoid collisions.

Two lateral sensor bars hold the ultra-sound transducers, the laser scanner, wires and a plastic box containing the interface board. Thus, the IntellWheels hardware framework can be easily attached to standard power wheelchairs from several different manufacturers to convert them into intelligent wheelchairs. Sensors bars are made of aluminium, which provides a good compromise between weight and robustness. Its black color makes the set more discreet, and is consistent with original lines of the wheelchair. The assemblage of the sensors bars do not interfere with the normal battery removal or wheelchair folding, operations usually required to facilitate the transportation of several wheelchair models.

The general configuration of sensors is shown in Figure 2. Lateral ultra-sound sensors (S3-S6 and S11-S14) are located $22 \mathrm{~cm}$ apart from each other and were assembled directly in the sensor bar. Front and rear sensors (S1, S2, S7, S8, S9, S10, S15 and S16) were assembled in a a special rounded tip designed to fit in both extremities of the sensor bars, and are headed with a $45^{\circ}$ difference. This configuration allows a safety perimeter, in which objects are always in the wheelchair field of view, that extends from $27 \mathrm{~cm}$ until $80 \mathrm{~cm}$. The figure also depicts the positioning of the laser scanner (L1) in the left sensor bar. Figure 3 identifies the location of the components of the hardware framework in the IntellWheels prototype. 


\section{RESUlts}

In order to evaluate the extent of the visual/ergonomic changes, we conducted a public opinion poll about the visual appearance of several intelligent wheelchair prototypes. In the survey, respondents were invited to express their level of agreement to fourteen statements through a typical five-level Likert scale ( 1 = Strongly disagree, $2=$ Disagree, $3=$ Neither agree nor disagree, $4=$ Agree, $5=$ Strongly agree).

The assessment was answered by 128 individuals, of which $43.8 \%$ males and $56.3 \%$ females, with a mean of age of 24.2 years old $(s t d=7.26)$. Respondents were selected by convenience, and are composed essentially by Master and PhD students, with 32\% having high school diplomas, 39\% Bachelor degrees, 25\% Master degrees and 3.1\% Doctors degrees. The majority of the sample, $54.7 \%$, is composed by subjects with no direct relation with physically disabled people, while $31.3 \%$ were health care professionals, $12.5 \%$ were relatives or friends of wheelchairs users and $1.6 \%$ were users of manual wheelchairs. The lower proportion of people with disabilities may not affect the validity of this study since the object under analysis, the public opinion about the visual appearance of intelligent wheelchair prototypes, is not determined by the condition of the subjects.

The questionnaire was composed of two parts. The goal of the first part was to evaluate the visual appearance of IntellWheels comparatively to other 9 intelligent wheelchair prototypes. Respondents were asked to express their level of agreement with the statement:

The addition of sensors and other hardware devices had visual/ergonomic impact on the wheelchair (e.g. changed the normal appearance/usage of the Wheelchair)

based on pictures of the intelligent wheelchair prototypes of the SmartChair, EISLAB, University of Shiga, University of Texas, IntellWheels, MIT, Robochair, Sharioto, SENA and FRIEND II. Results of the first part of the questionnaire are depicted in the Figure 4a. An analysis within subject performed with the Wilcoxon signed rank test (Table I) provided statistical evidence that, compared to each one of the other nine projects, IntellWheels presented the lowest change in the normal appearance of the wheelchair.

In the second part, the goal was to assess the visual impact of the modifications performed in the wheelchair as a whole, as well as the visual changes introduced by specific hardware devices (display, sensor bars and other hardware devices). Respondents were presented to a picture of the original powered wheelchair and of the IntellWheels prototype, and requested to express their level of agreement with the statements:

In comparison with the original powered wheelchair, global visual/ changes of the IntellWheels prototype are small.

In comparison with the original powered wheelchair, visual changes introduced by the display are small.

In comparison with the original powered wheelchair, visual changes introduced by the sensor bars are small.

In comparison with the original powered wheelchair, visual changes introduced by the computer and other hardware are small.
TABLE I: Wilcoxon signed rank test: comparison between the visual impact of IntellWhells with other IW prototypes.

\begin{tabular}{l||cc}
\hline & $\mathrm{Z}$ & $\mathrm{p}$ \\
\hline IntellWhells - SmartChair & -5.622 & $<0.001$ \\
IntellWhells - Robochair & -2.403 & 0.008 \\
IntellWhells - Shiga & -2.756 & 0.003 \\
IntellWhells - Texas & -5.547 & $<0.001$ \\
IntellWhells - MIT & -2.306 & 0.010 \\
IntellWhells - EISLAB & -4.566 & $<0.001$ \\
IntellWhells - Sharioto & -2.446 & 0.007 \\
IntellWhells - SENA & -5.613 & $<0.001$ \\
IntellWhells - FRIENDII & -6.218 & $<0.001$ \\
\hline
\end{tabular}

TABLE II: Wilcoxon test: Opinions regarding four items were compared to the neutral hypothesis $(3=$ Neither agree nor disagree).

\begin{tabular}{l||cc}
\hline & $\mathrm{Z}$ & $\mathrm{p}$ \\
\hline Hypothesis - Global & -6.036 & $<0.001$ \\
Hypothesis - Display & -6.319 & $<0.001$ \\
Hypothesis - Sensor bar & -4.217 & $<0.001$ \\
Hypothesis - Other hardware & -7.051 & $<0.001$ \\
\hline
\end{tabular}

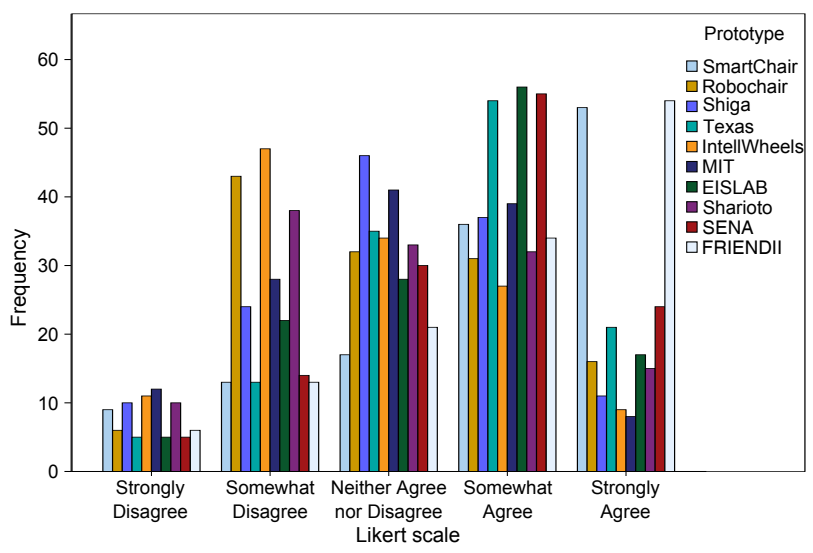

(a)

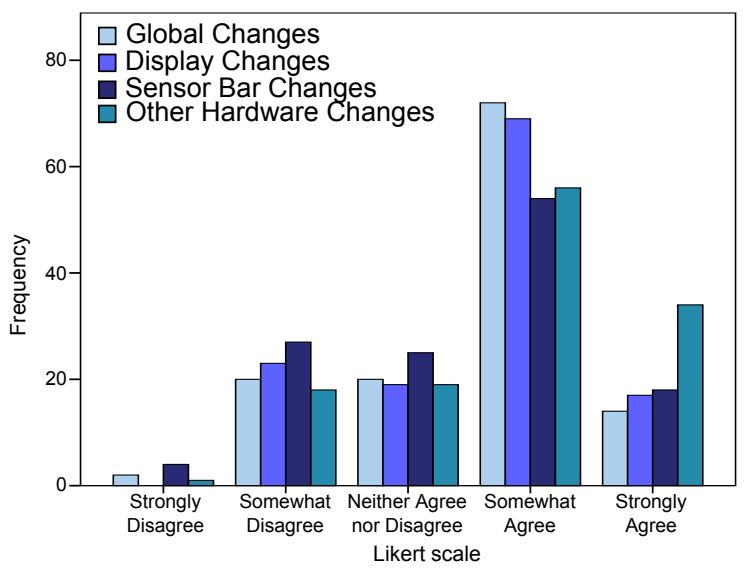

(b)

Fig. 4: Responses indicating the user opinion regarding: (a) each one of the ten intelligent wheelchair prototypes, and (b) four characteristics of the IntellWheels prototype.

Figure $4 \mathrm{~b}$ depicts the responses of the second part of the questionnaire. An analysis within subject was performed with the Wilcoxon signed rank test (Table II) by comparing the opinions regarding the four statements to the neutral hypothesis ( $3=$ Neither agree nor disagree). At $\alpha=0.05$ level of significance, there exists enough evidence to conclude that both 
the display, sensor bars and other hardware devices had only a small visual impact. Further statistical results indicate that the IntellWheels prototype was able to keep the overall aspect of the original wheelchair.

\section{CONCLUSION}

In this paper we presented a flexible hardware framework, which design concerns with minimizing the visual and ergonomic impacts of the addition of sensing and computational capabilities. Its modular architecture enables an easy integration of distinct hardware and software methodologies and facilitates the conversion of ordinary powered wheelchairs into intelligent wheelchairs with minor changes. The proposed hardware framework presented a cost effective solution for assisting severely impaired individuals, with estimated cost around $2.000,00 €($ the same price of a simple powered wheelchair).

In addition, we demonstrate that despite the assemblage of the several hardware devices, the accessibility to the wheelchair was not compromised. The assessment of the IntellWheels visual appearance indicated that not only the prototype presented the lowest visual impact between ten other intelligent wheelchair prototypes, but also that its overall aspect is similar to the original powered wheelchair. Such result validate the design of the IntellWheels prototype and contribute to increase the acceptance of assistive robotics by the general population.

\section{ACKNOWLEDGMENT}

This work was funded by the ERDF European Regional Development Fund through the COMPETE Programme and by National Funds through FCT - Portuguese Foundation for Science and Technology within project INTELLWHEELS, RIPD/ADA/109636/2009. The first and third authors would like to acknowledge FCT for the PhD Grants ref. SFRH/BD/60727/2009 and SFRH/BD/44541/2008.

\section{REFERENCES}

[1] M. A. Jones, I. R. McEwen, and L. Hansen, "Use of power mobility for a young child with spinal muscular atrophy," Physical Therapy, vol. 83, no. 3, pp. 253-262, 2003.

[2] R. C. Simpson, E. F. LoPresti, and R. A. Cooper, "How many people would benefit from a smart wheelchair?" Journal of Rehabilitation Research and Development, vol. 45, no. 1, pp. 53-71, 2008.

[3] L. Fehr, W. E. Langbein, and S. B. Skaar, "Adequacy of power wheelchair control interfaces for persons with severe disabilities: A clinical survey," Journal of Rehabilitation Research and Development, vol. 37, no. 3, pp. 353-360, May/June 2000.

[4] R. A. Braga, M. Petry, L. P. Reis, and A. P. Moreira, "Intellwheels: Modular development platform for intelligent wheelchairs," Journal of Rehabilitation Research and Development, vol. 48, no. 9, pp. 10611076, December 2011.

[5] S. P. Parikh, V. Grassi Jr, V. Kumar, and J. Okamoto Jr, "Integrating human inputs with autonomous behaviors on an intelligent wheelchair platform," Intelligent Systems, IEEE, vol. 22, no. 2, pp. 33-41, April 2007.

[6] S. Rnnbck, J. Piekkari, K. Hyypp, T. Berglund, and S. Koskinen, "A semi-autonomous wheelchair towards user-centered design," in Computers Helping People with Special Needs, ser. LNCS. Berlin: Springer Berlin Heidelberg, 2006, vol. 4061, pp. 701-708.

[7] J. Gonzalez, A. J. Muaeoz, C. Galindo, J. A. Fernandez-Madrigal, and J. L. Blanco, "A description of the sena robotic wheelchair," in IEEE Mediterranean Electrotechnical Conference - MELECON, Malaga, Spain, 16-19 May 2006, pp. 437-440.
[8] T. Hamagami and H. Hirata, "Development of intelligent wheelchair acquiring autonomous, cooperative, and collaborative behavior," in IEEE International Conference on Systems, Man \& Cybernetics, vol. 1-7, Hague, Netherlands, 10 -13 October 2004, pp. 3525-3530.

[9] I. Volosyak, O. Ivlev, and A. Graser, "Rehabilitation robot friend iithe general concept and current implementation," in 9th International Conference on Rehabilitation Robotics - ICORR. Chicago, USA: IEEE, 28 June-1 July 2005, pp. 540-544.

[10] P. Jia, H. Hu, T. Lu, and K. Yuan, "Head gesture recognition for handsfree control of an intelligent wheelchair," Journal of Industrial Robot, vol. IV, no. 1, pp. 60-68, 2007.

[11] S. Hemachandra, T. Kollar, N. Roy, and S. Teller, "Following and interpreting narrated guided tours," in IEEE International Conference on Robotics and Automation - ICRA. Changhai, China: IEEE, 9-11 May 2011, pp. 2574-2579.

[12] D. Vanhooydonck, E. Demeester, M. Nuttin, and H. Van Brussel, "Shared control for intelligent wheelchairs: an implicit estimation of the user intention," in International Workshop on Advances in Service Robotics - ASER03. Bardolino, Italy: Citeseer, 13-15 March 2003, pp. 176-182.

[13] T. Yasuda, K. Nakamura, A. Kawahara, and K. Tanaka, "Neural network with variable type connection weights for autonomous obstacle avoidance on a prototype of six-wheel type intelligent wheelchair," International Journal of Innovative Computing, Information and Control, vol. 2, no. 5, pp. 1165-1177, October 2006.

[14] G. Pires and U. Nunes, "A wheelchair steered through voice commands and assisted by a reactive fuzzy-logic controller," Journal of Intelligent and Robotic Systems, vol. 34, no. 3, pp. 301-314, July 2002.

[15] S. Gulati and B. Kuipers, "High performance control for graceful motion of an intelligent wheelchair," in IEEE International Conference on Robotics and Automation - ICRA'08. Pasadena, USA: IEEE, 19-23 May 2008, pp. 3932-3938.

[16] H. Soh and Y. Demiris, "Towards early mobility independence: An intelligent paediatric wheelchair with case studies," in IEEE/RSJ International Conference on Intelligent Robots and Systems. Workshop on Progress, Challenges and Future Perspectives in Navigation and Manipulation Assistance for Robotic Wheelchairs, Vila Moura, Portugal, 2012, pp. 1-7.

[17] I. Iturrate, J. M. Antelis, A. Kubler, and J. Minguez, "A noninvasive brain-actuated wheelchair based on a p300 neurophysiological protocol and automated navigation," IEEE Transactions on Robotics, vol. 25, no. 3, pp. 614-627, 2009.

[18] M. Petry, A. P. Moreira, L. P. Reis, and R. Rossetti, "Intelligent wheelchair simulation: Requirements and architectural issues," in International Conference on Mobile Robots and Competitions, Lisbon, Portugal, 6 April 2011, pp. 102-107.

[19] B. M. Faria, L. P. Reis, and N. Lau, "Cerebral palsy eeg signals classification: Facial expressions and thoughts for driving an intelligent wheelchair," in International Conference on Data Mining - Biological Data Mining and its Applications in Healthcare Workshop, Brussels, Belgium, 10-13 December 2012, pp. 33-40.

[20] B. M. Faria, S. Vasconcelos, L. P. Reis, and N. Lau, "Evaluation of distinct input methods of an intelligent wheelchair in simulated and real environments: A performance and usability study," Assistive Technology: The Official Journal of RESNA, vol. 25, no. 2, pp. 88-98, September 2012.

[21] _ "A methodology for creating intelligent wheelchair users' profiles," in International Conference on Agents and Artificial Intelligence - ICAART. Vila Moura, Portugal: ICAART, 6-8 February 2012, pp. 171-179.

[22] R. A. M. Braga, M. Petry, A. P. Moreira, and L. P. Reis, "Concept and design of the intellwheels development platform for intelligent wheelchairs," in Informatics in Control, Automation and Robotics, ser. LNEE, J. A. Cetto, J.-L. Ferrier, and J. Filipe, Eds. Heidelberg: Springer-Verlag, 2009, vol. 37, pp. 191-203.

[23] R. A. M. Braga, M. Petry, L. P. Reis, and A. P. Moreira, "Plataform for intelligent wheelchairs using multi-level control and probabilistic motion model," pp. 833-838, July 21232008. 